\title{
Six new species of Handaoia Seyrig, 1952 (Hymenoptera, Ichneumonidae, Phygadeuontinae): the first to be described from the New World
}

\author{
Santiago BORDERA ${ }^{1, *} \&$ Gavin R. BROAD ${ }^{2}$ \\ ${ }^{1}$ Departamento de Ciencias Ambientales y Recursos Naturales, University of Alicante, \\ San Vicente del Raspeig, E-03690 Alicante, Spain. \\ ${ }^{2}$ Department of Life Sciences, the Natural History Museum, Cromwell Road, London SW7 5BD, UK. \\ *Corresponding author: s.bordera@ua.es \\ 2Email: g.broad@nhm.ac.uk \\ 1 (1) https://orcid.org/0000-0002-1713-4043 \\ 2 이 https://orcid.org/0000-0001-7223-5333 \\ ${ }^{1}$ urn:lsid:zoobank.org:author:CE4BC30D-26D7-4F70-BAAC-3A6FBDF49AFD \\ ${ }^{2}$ urn:1sid:zoobank.org:author:D06689DE-526F-4CFA-8BEB-9FB38850754A
}

\begin{abstract}
Handaoia Seyrig, 1952 is a small genus of Phygadeuontinae currently represented by eleven described species from Madagascar, Tanzania and Europe, and can be recognized by the combination of the distally expanded and ventrally flattened antennal flagellum, complete posterior transverse carina of the mesosternum, isolated 'pit' (episternal scrobe) in the mesopleuron, and a single bulla in fore wing vein $2 m-c u$. Most species have a distinctive combined area basalis and area superomedia on the propodeum. The following six new species from Central and South America are described and illustrated: H. cuscoensis Bordera sp. nov. from Peru, $H$. fritzi sp. nov. from Brazil, H. mercedensis Bordera sp. nov. from Peru, H. plaumanni sp. nov. from Brazil, H. ruizcancinoi Bordera sp. nov. from Mexico, and H. urceus sp. nov. from Brazil. A key to the New World species is provided.
\end{abstract}

Keywords. Taxonomy, parasitoid, Darwin wasps, new species, key.

Bordera S. \& Broad G.R. 2021. Six new species of Handaoia Seyrig, 1952 (Hymenoptera, Ichneumonidae, Phygadeuontinae): the first to be described from the New World. European Journal of Taxonomy xxx: xxx-yyy. https://doi.org/10.5852/ejt.2021.757.1415

\section{Introduction}

Handaoia Seyrig, 1952 is a small genus of Phygadeuontinae Förster, 1869 (sensu Santos 2017), currently represented by eleven described species (Yu et al. 2016): seven from Madagascar (Seyrig 1952), one from Tanzania (Szépligeti 1908) and two from Central and Northern Europe (Horstmann 1979, 1981). Undescribed species have also been reported from the Philippines, Japan, South Africa, Peru (Townes 1970) and Australia (Gauld 1984). Townes (1970) classified Handaoia in the subtribe 
Chiroticina Townes, 1970 (Cryptinae, Phygadeuontini). Nothing is known about the biology of any species of Handaoia, although by analogy with other Phygadeuontinae, they can be expected to be idiobiont ectoparasitoids of larvae or pupae of holometabolous insects.

While studying specimens from three collections with extensive Neotropical Phygadeuontinae holdings, the Natural History Museum (London, UK), American Entomological Institute (Utah, USA) and Colección Entomológica de la Universidad de Alicante (Spain), we discovered six new species of Handaoia from Central and South America. As the genus had previously been reported only from Peru within the Neotropical region (Townes 1970), and only represented by undescribed species, these specimens significantly extend the known distribution and diversity of Handaoia. The aim of this paper is to describe and illustrate these new species, and to provide a key to the New World.

\title{
Material and methods
}

Material deposited in the following institutions has been studied:

AEIC = American Entomological Institute Collection, Utah, USA

CEUA $=$ Colección Entomológica de la Universidad de Alicante, Alicante, Spain

NHMUK $=$ Natural History Museum, London, UK

Morphological terminology follows Broad et al. (2018) with surface microsculpture descriptions based on Eady (1968). Layer (extended focus) photographs of $H$. fritzi sp. nov., $H$. plaumanni sp, nov. and H. urceus sp. nov. were taken at NHMUK using a Canon EOS 5DSR digital camera with a Canon MP-E $65 \mathrm{~mm}$ Macro Lens attached to a StackShot Macro Rail system controlled by Helicon Remote and Helicon Focus ver. 3.6.6W. Layer photos of H. cuscoensis Bordera sp. nov., H. mercedensis Bordera sp. nov. and H. ruizcancinoi Bordera sp. nov. were taken at the University of Alicante using a Canon EOS 70D digital camera with the same macro lens and system.

\section{Results}

\author{
Class Insecta Linnaeus, 1758 \\ Order Hymenoptera Linnaeus, 1758 \\ Superfamily Ichneumonoidea Latreille, 1802 \\ Family Ichneumonidae Latreille, 1802 \\ Subfamily Phygadeuontinae Förster, 1869
}

Genus Handaoia Seyrig, 1952

Handaoia Seyrig, 1952: 26 (type species: Handaoia spinosa Seyrig, 1952, by original designation).

\section{Diagnosis}

Handaoia can be recognized and separated from other Phygadeuontinae genera by the combination of the distally expanded and ventrally flattened antennal flagellum, complete posterior transverse carina of the mesosternum, isolated 'pit' (episternal scrobe) in the mesopleuron, and a single bulla in fore wing vein $2 m-c u$. The following more complete diagnosis is modified from Townes (1970), who included Handaoia in a key to the genera of 'Chiroticina'. Townes' definition applies equally well to the Afrotropical species originally included as well as to the Neotropical and Palaearctic species: flagellum of female antenna fusiform (expanded beyond the middle and flattened ventrally) with a median white ring; genal carina reaching base of mandible; mandibular teeth subequal; maxillary palp long, reaching beyond centre of mesosternum; pronotum with two dorsal pits separated by weak longitudinal carina, usually flanked laterally by 3-4 longitudinal carinae; epomia absent; median lobe of mesoscutum without 
median longitudinal groove; scutellum without lateral carinae; mesopleural impression (episternal scrobe) ventral to speculum as an isolated pit, some distance from mesopleural furrow; posterior transverse carina of mesosternum complete; fore wing with areolet pentagonal, its outer side open (vein $3 r s-m$ missing), vein $2 m$-cu with one wide bulla; area superomedia always separated from area petiolaris and usually confluent with area basalis; laterotergites of metasomal tergites 2 and 3 not separated or only weakly separated from tergite. Small species, body length $2-5 \mathrm{~mm}$.

\section{Key to the New World species of Handaoia}

1. Apophysis of propodeum high, parallel sided or subtriangular, with blunt tip (Figs 2F, 4G, 7H, arrows)

- Apophysis of propodeum as a low transverse rounded crest, or slightly pointed (Figs 3G, 5I, 8I, arrows)

2. Area externa of propodeum confluent with area dentipara (Fig. 1A). Juxtacoxal carina absent (Fig. 2C). First and second flagellomeres about 5.6 and $6.5 \times$ as long as wide respectively. Hind coxa tinged with white (Fig. 2A)

H. cuscoensis Bordera sp. nov.

- Area externa of propodeum separated from area dentipara by strong carina (Fig. 1C, E). Juxtacoxal carina present, usually strong (Figs 4F, 7G). First and second flagellomeres about 4-5 and 3.2-5.4× as long as wide, respectively. Hind coxa entirely dark brown (Figs 4A, 7A)

3. Fore wing with well-defined transverse dark brown band (Fig. 4A). Inner side of area externa $4.7 \times$ as long as inner side of area dentipara (Fig. 1C). Juxtacoxal carina strong, irregular, joining submetapleural carina at its mid-length (Fig. 4F). Tergite I about $1.8 \times$ as long as its maximum width. First and second flagellomeres about 5 and $5.4 \times$ as long as wide respectively. Flagellum dark brown (except white band), with first and second flagellomeres light brown (Fig. 4AB)

H. mercedensis Bordera sp. nov.

- Fore wing evenly infuscate (Fig. 7A). Inner side of area externa $7.0 \times$ as long as inner side of area dentipara (Fig. 1E). Juxtacoxal carina weak but complete, joining anterior part of submetapleural carina (Fig. $7 \mathrm{G}$ ). Tergite I about $2.4 \times$ as long as its maximum width. First and second flagellomeres 4.1 and $3.3 \times$ as long as wide respectively. Flagellum dark brown (except white band), with only first flagellomere light brown (Fig. 7A)

H. ruizcancinoi Bordera sp. nov.

4. Area externa of propodeum confluent with area dentipara (Fig. 1D). Juxtacoxal carina absent (Fig. 5H). Sternaulus very short and weak, reaching at most $0.3 \times$ the length of the mesopleuron (Fig. 5E). Head coarsely granulate (Fig. 5B-C)

H. plaumanni sp. nov.

- Area externa of propodeum separated from area dentipara by a strong carina (Fig. 1B, F). Juxtacoxal carina present (Figs $3 \mathrm{~F}, 8 \mathrm{H}$, arrows). Sternaulus deep, reaching at least $0.5 \times$ the length of the mesopleuron (Figs 3D, 8G). Head finely granulate

5. Inner side of area externa 3.0-3.4 $\times$ as long as inner side of area dentipara (Fig. 1B); inner side of area dentipara (section of the lateromedian longitudinal carina) $0.4-0.5 \times$ the length of the outer side (section of the lateral longitudinal carina) (Fig. 1B). Lateromedian longitudinal carina of first metasomal tergite weak, reaching at most $0.6 \times$ the length of the tergite, or sometimes inconspicuous (Fig. 1B). Malar space $1.15-1.25 \times$ as long as basal mandibular width (Fig. 3E). Tergite II smooth and shiny (Fig. 3B). Clypeus 2.1-2.2 $\times$ as wide as high (Fig. 3E)

H. fritzi sp. nov.

- Inner side of area externa about $8.0 \times$ as long as inner side of area dentipara (Fig. 1F); inner side of area dentipara $0.25 \times$ the length of the outer side (Fig. 1F). Lateromedian longitudinal carina of tergite I strong, reaching $0.8 \times$ the length of the tergite (Fig. 1F). Malar space $1.4 \times$ as long as mandible base (Fig. 8B). Tergite II finely granulate (Fig. 8D). Clypeus $1.6 \times$ as wide as high (Fig. 8B)

H. urceus sp. nov. 


\section{Handaoia cuscoensis Bordera sp. nov. urn:1sid:zoobank.org:act:49A19681-3B7F-4E6F-B95B-4E6847D1C5AF}

Figs $1 \mathrm{~A}, 2$, $9 \mathrm{~B}$

\section{Diagnosis}

Handaoia cuscoensis Bordera sp. nov. can be distinguished from all other New World species by the combination of the following characters: apophysis of propodeum high, subtriangular with blunt tip (Fig. 2F, arrow); juxtacoxal carina absent (Fig. 2C); area externa of propodeum confluent with area dentipara (Fig. 1A); hind coxa tinged with white (Fig. 2A).

\section{Etymology}

The name of the species refers to the Department of Cusco (Peru), the type locality region.

\section{Material examined}

Only known from the holotype female.

\section{Holotype}

PERU • + ; Quincemil, 750 m nr. Marcapata; 10-15 Nov. 1962; Luis Peña leg.; AEIC.

\section{Description}

\section{Female}

Measurements. Body length (without ovipositor) $3.8 \mathrm{~mm}$. Fore wing $2.9 \mathrm{~mm}$ long.

HEAD. Very finely and densely granulate, matt, distinctly narrowed behind eyes, occiput very concave centrally in dorsal view (Fig. 2E); gena about $0.15 \times$ length of eye in dorsal view; posterior ocellus separated from eye by $1.43 \times$ its own maximum diameter; distance between posterior ocelli $1.14 \times$ maximum ocellar diameter (Fig. 2E); occipital carina gently V-shaped medially; malar space $1.33 \times$ basal width of mandible; face moderately swollen medially (Fig. 2B); clypeus very weakly convex, almost flat, finely and slightly granulate with scattered punctures, its apex weakly rounded, about $2.3 \times$ as wide as high; mandible slightly tapered to apex, ventral tooth of mandible a little shorter than upper tooth; maxillary palp reaching at most $3 / 4$ length of mesosternum; flagellum widened in middle, tapered towards apex, flagellomeres 8-19 flattened ventrally (Fig. 1A); flagellum with 20 segments, lengththickness ratios: $1^{\text {st }}$ segment $=5.64,2^{\text {nd }}=6.55$ and $10^{\text {th }}=1.08$.

Mesosoma. Very finely and densely granulate, matt (Fig. 2D); mesoscutum conspicuously convex, notauli absent (Fig. 2E); scutellum moderately convex, without lateral carinae; sternaulus very short and weak, as a wide V-shaped depression anteriorly, reaching at most $0.3 \times$ length of mesopleuron (Fig. 2D); juxtacoxal carina barely indicated anteriorly (Fig. 2C); propodeum (Fig. 1A) with mostly weak carinae; area superomedia confluent with area basalis and separated from area petiolaris; area externa and area dentipara confluent; area spiracularis confluent with area lateralis; area dentipara section of lateral longitudinal carina very weak, partly absent; posterior transverse carina strong, forming elevate apophysis joining lateral longitudinal carina, apophysis subtriangular, with blunt tip (Fig. 2D, F, arrow). Length of hind femur about $4.4 \times$ its height. Hind wing with vein $c u-a 0.73$ as long as abscissa of $C U$ between $M$ and $c u-a$.

Metasoma. Tergite I (Figs 1A, 2G) finely granulate and very weakly strigose laterally, matt, $2.5 \times$ as long as its maximum width; lateromedian longitudinal carina absent; lateral longitudinal carina complete; tergite II (Fig. 2G) shallowly granulate, shiny, glabrous; remaining tergites (Fig. 2G) smooth and shiny with inconspicuous dense setiferous punctures. Ovipositor sheath about $0.4 \times$ as long as hind tibia. 
Colour. Head (Fig. 2A-B, D-E) light orange; antenna with scape and pedicel light brown, flagellum dark brown, distal part of flagellomere 4 to flagellomere 7 dorsally white (Fig. 2A). Mesosoma (Fig. 2D-
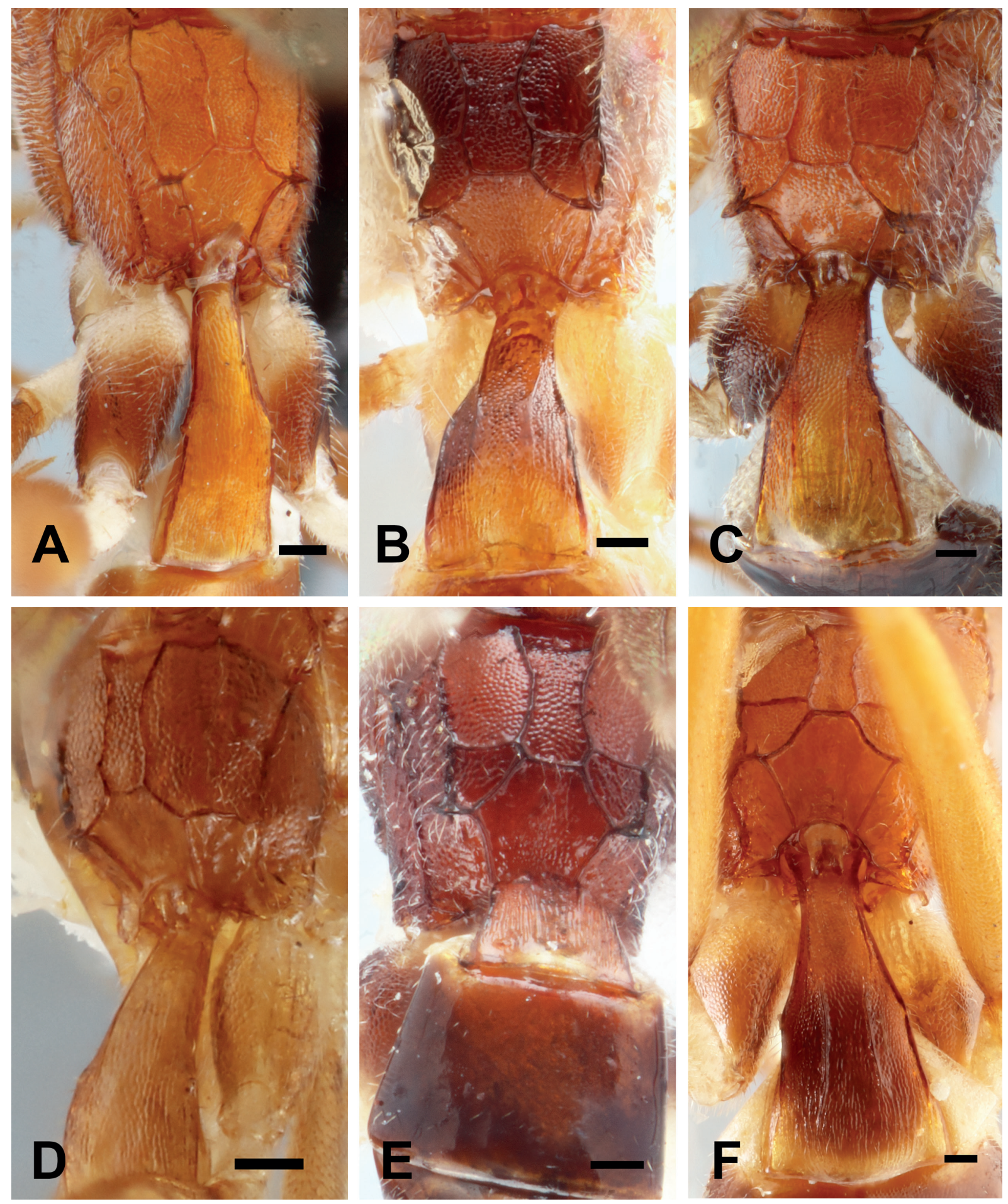

Fig. 1. Propodeum and tergite I of Handaoia spp., q $q$. A. H. cuscoensis Bordera sp. nov., holotype (AEIC). B. H. fritzi sp. nov., holotype (NHMUK). C. H. mercedensis Bordera sp. nov., holotype (CEUA). D. H. plaumanni sp. nov., holotype (NHMUK). E. H. ruizcancinoi Bordera sp. nov., holotype (CEUA). F. H. urceus sp. nov., holotype (NHMUK). Scale bars: $0.1 \mathrm{~mm}$. 
E) light orange. Metasoma (Fig. 2G) mostly brown, tergites I-II and anterior part of IV light orange. Legs (Fig. 2A) mostly light orange, distal tarsomeres, dark brown, fore and mid coxae, trochanters and trochantelli white, hind coxa white proximally, dark brown distally. Wings (Fig. 2A) slightly evenly infuscate.

Male

Unknown.
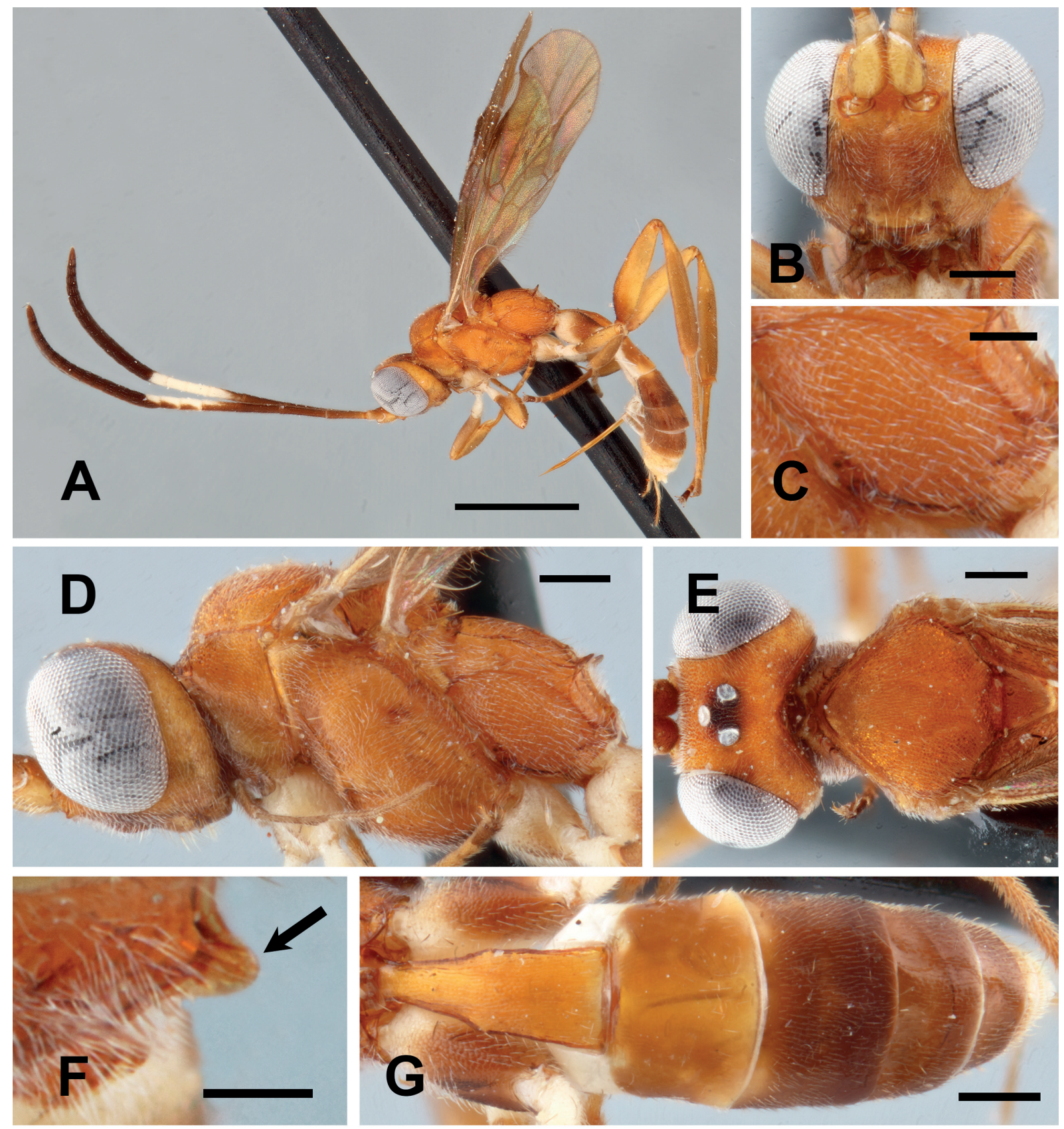

Fig. 2. Morphology of Handaoia cuscoensis Bordera sp. nov., holotype, $q$ (AEIC). A. Habitus, lateral view. B. Head, front view. C. Metapleuron. D. Head and mesosoma, lateral view. E. Head and mesoscutum, dorsal view. F. Propodeum (arrow: propodeal apophysis). G. Metasoma, dorsal view. Scale bars: $A=1 \mathrm{~mm}$; B, D-E, $G=0.2 \mathrm{~mm} ; C, F=0.1 \mathrm{~mm}$. 


\section{Handaoia fritzi sp. nov. urn:1sid:zoobank.org:act:3FE16228-86F1-4A8C-8FA0-227999E65A4F}

Figs 1B, 3, 9B

\section{Diagnosis}

Handaoia fritzi sp. nov. can be distinguished from all other New World species by the combination of the following characters: apophysis of propodeum as a low transverse rounded or slightly pointed triangular crest (Fig. 3G, arrow); juxtacoxal carina strong, irregular, joining submetapleural carina at anterior $0.3-0.4$ (Fig. 3F, arrow); area externa of propodeum separated from area dentipara by strong carina (Fig. 1B); inner side of area externa 3.0-3.4 $\times$ as long as inner side of area dentipara (Fig. 1B); inner side of area dentipara $0.46-0.5 \times$ the length of outer side (Fig. 1B); tergite II smooth and shiny (Fig. 3B).

\section{Etymology}

This species is named in honour of Dr Fritz Plaumann, illustrious botanist and entomologist based in Brazil, who collected the type material. A noun in genitive case.

\section{Material examined}

Known from five females.

\section{Holotype}

BRAZIL • + ; Nova Teutonia; 27ํ1' S, 52²3' W; 26 Aug. 1937; F. Plaumann leg.; B.M. 1937-748; B.M. Type Hym 3b.2871; NHMUK010636381.

\section{Paratypes}

BRAZIL• 1 \%; same collection data as for holotype;Aug. 1935; B.M. 1937-47; NHMUK010636388• 1 q ; same collection data as for preceding; 28 May 1938; B.M. 1938-458; NHMUK010636387 • 1 क; same collection data as for preceding; 11 Aug. 1938; B.M. 1938-682; NHMUK010636396 • 1 o; same collection data as for preceding; 16 Sep. 1938; B.M. 1938-682; NHMUK010636383.

\section{Description}

\section{Female}

MeAsurements. Body length (without ovipositor) 3.3-4.1 mm. Fore wing 2.7-3.3 mm long.

HEAD. Finely granulate, matt, distinctly narrowed behind eyes, occiput very concave centrally in dorsal view (Fig. 3C); gena $0.2-0.25 \times$ length of eye in dorsal view; posterior ocellus separated from eye by $0.75-1.25 \times$ its own maximum diameter; distance between posterior ocelli $0.75-1.0 \times$ maximum ocellar diameter (Fig. 3C); occipital carina gently V-shaped medially (Fig. 3B); malar space 1.15-1.25 $\times$ basal width of mandible; face slightly swollen medially; clypeus (Fig. 3E) weakly convex, faintly granulate dorsally, smooth and shiny ventrally, with scattered punctures, its apex almost straight, $2.1-2.2 \times$ as wide as high; mandible slightly tapered to apex, ventral tooth of mandible approximately same length as upper tooth; maxillary palp relatively short, reaching at most $2 / 3$ length of mesosternum; flagellum widened in middle, tapered towards apex, flagellomeres 8-17 flattened ventrally; flagellum with 19-20 segments, length-thickness ratios: $1^{\text {st }}$ segment $=4.74-5.0,2^{\text {nd }}=5.0-5.28$ and $10^{\text {th }}=1.0$.

Mesosoma. Finely granulate and matt (Fig. 3B-D); mesoscutum convex, notauli weak anteriorly, reaching about 0.3 distance to scuto-scutellar groove, sometimes inconspicuous (Fig. 3C); scutellum moderately convex, without lateral carinae; sternaulus moderately deep, reaching at least middle of mesopleuron (Fig. 3D); juxtacoxal carina strong, irregular, joining submetapleural carina at anterior 0.3-0.4 (Fig. 3F, arrow); propodeum (Fig. 1B) with strong and conspicuous carinae; area superomedia 

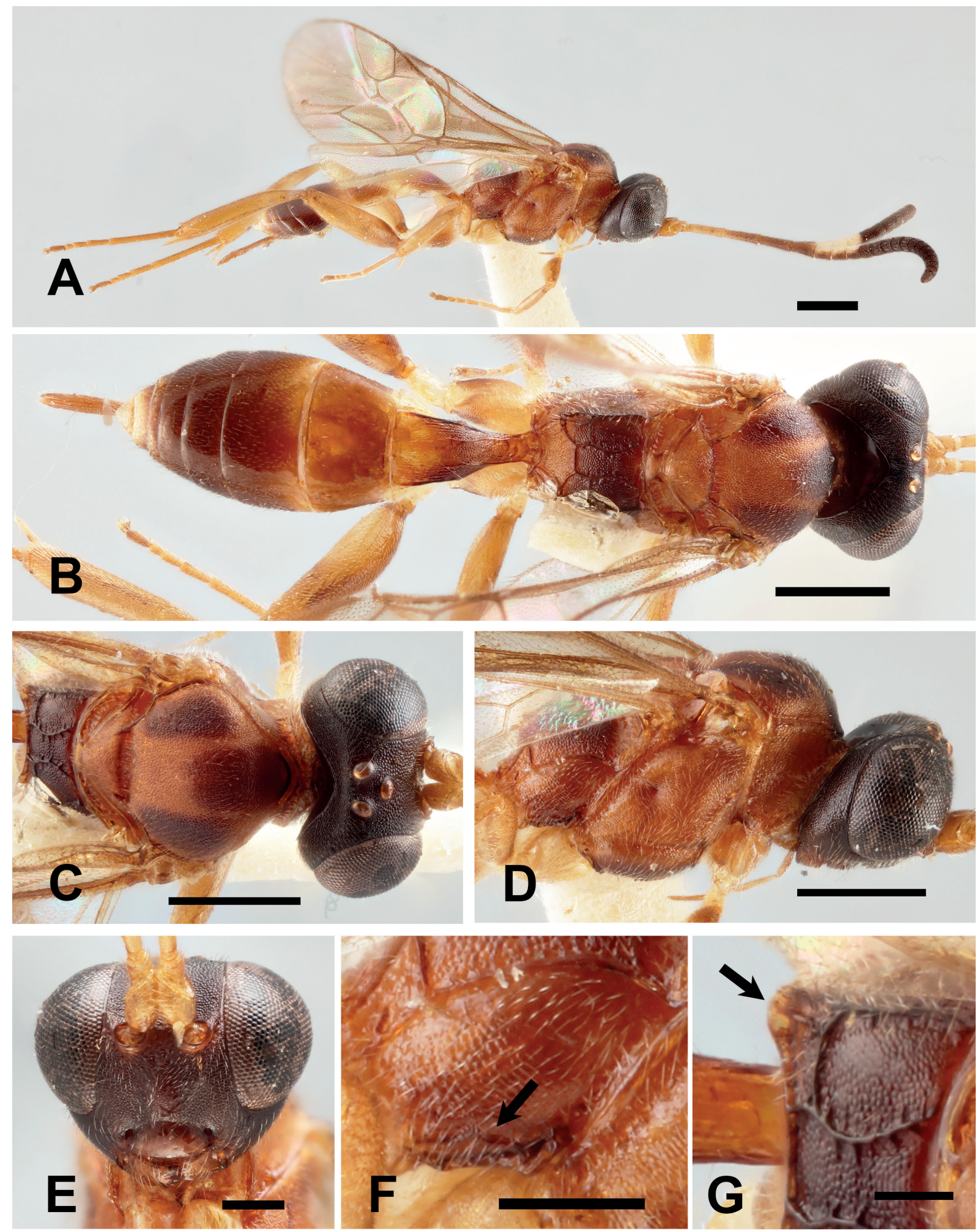

Fig. 3. Morphology of Handaoia fritzi sp. nov., holotype, $q$ (NHMUK010636396). A. Habitus, lateral view. B. Habitus, dorsal view. C. Head and mesoscutum, dorsal view. D. Head and mesosoma, lateral view. E. Head, front view. F. Metapleuron (arrow: juxtacoxal carina). G. Propodeum (arrow: propodeal apophysis). Scale bars: A-D $=0.5 \mathrm{~mm} ; \mathrm{E}-\mathrm{F}=0.2 \mathrm{~mm} ; \mathrm{G}=0.1 \mathrm{~mm}$. 
confluent with area basalis and separated from area petiolaris; inner side of area externa about 3.0-4.0 $\times$ as long as inner side of area dentipara; inner side of area dentipara $0.46-0.5 \times$ length of outer side; area spiracularis confluent with area lateralis; posterior transverse carina strong, forming a low and wide rounded or slightly subtriangular crest joining lateral longitudinal carina (Fig. 3G, arrow). Length of hind femur 4.0-4.7 $\times$ its height. Hind wing with vein $c u-a 0.4-0.6 \times$ as long as abscissa of $C U$ between $M$ and $c u-a$.

Metasoma. Tergite I (Figs 1B, 3B), finely granulate, matt, $1.8-2.0 \times$ as long as its maximum width; postpetiole laterally and posteriorly tending to fine strigosity; lateromedian longitudinal carina weak, reaching at least $0.6 \times$ length of tergite; lateral longitudinal carina weak, complete or absent posterior to spiracles; tergite II (Fig. 3B) smooth and shiny, glabrous; remaining tergites (Fig. 3B) smooth and shiny with inconspicuous dense setiferous punctures. Ovipositor sheath $0.45-0.49 \times$ as long as hind tibia.

Colour. Head (Fig. 3A-E) dark brown, clypeus and mandibles reddish brown; palpi light brown; antenna with scape, pedicel and flagellomeres 1-4 light brown, darker dorsally, remaining flagellomeres dark brown, flagellomeres 4-7 dorsally white (Fig. 3A). Mesosoma (Fig. 3A-D) mostly dark orange; mesoscutum with wide dark brown longitudinal bands on central and lateral lobes; dorsal anterior part of propodeum dark brown. Metasoma (Fig. 3B) mostly brown, tergite I sometimes centrally tinged dark brown. Legs (Fig. 3A) light brown, distal tarsomeres dark brown, fore and mid coxae, part of hind coxa and all trochanters and trochantelli lighter. Wings (Fig. 3A) slightly infuscate.

Male

Unknown.

Handaoia mercedensis Bordera sp. nov. urn:1sid:zoobank.org:act:80A6A583-DB23-43D9-AF4A-4D16A6270918

Figs 1C, 4, 9B

\section{Diagnosis}

Handaoia mercedensis Bordera sp. nov. can be distinguished from all other New World species by the combination of the following characters: apophysis of propodeum conspicuously elevated, parallelsided with blunt tip (Fig. 4G, arrow); fore wing with well-defined transverse dark brown band (Fig. 4A); juxtacoxal carina strong, irregular, joining submetapleural carina at mid length (Fig. 4F, arrow); flagellum dark brown (except white band), with first and second flagellomeres light brown (Fig. 4A-B).

\section{Etymology}

The name refers to La Merced, municipality of Chanchamayo in the Department of Junin (Peru), where the holotype was collected.

\section{Material examined}

Known only from the holotype female.

\section{Holotype}

PERU • ; La Merced, Fundo La Génova; Trampa Malaise 3; 3-17 Jan. 2009; AECID 013484/07; CEUA.

\section{Description}

Female

MEAsurements. Body length (without ovipositor) $4.3 \mathrm{~mm}$. Fore wing $3.4 \mathrm{~mm}$ long. 

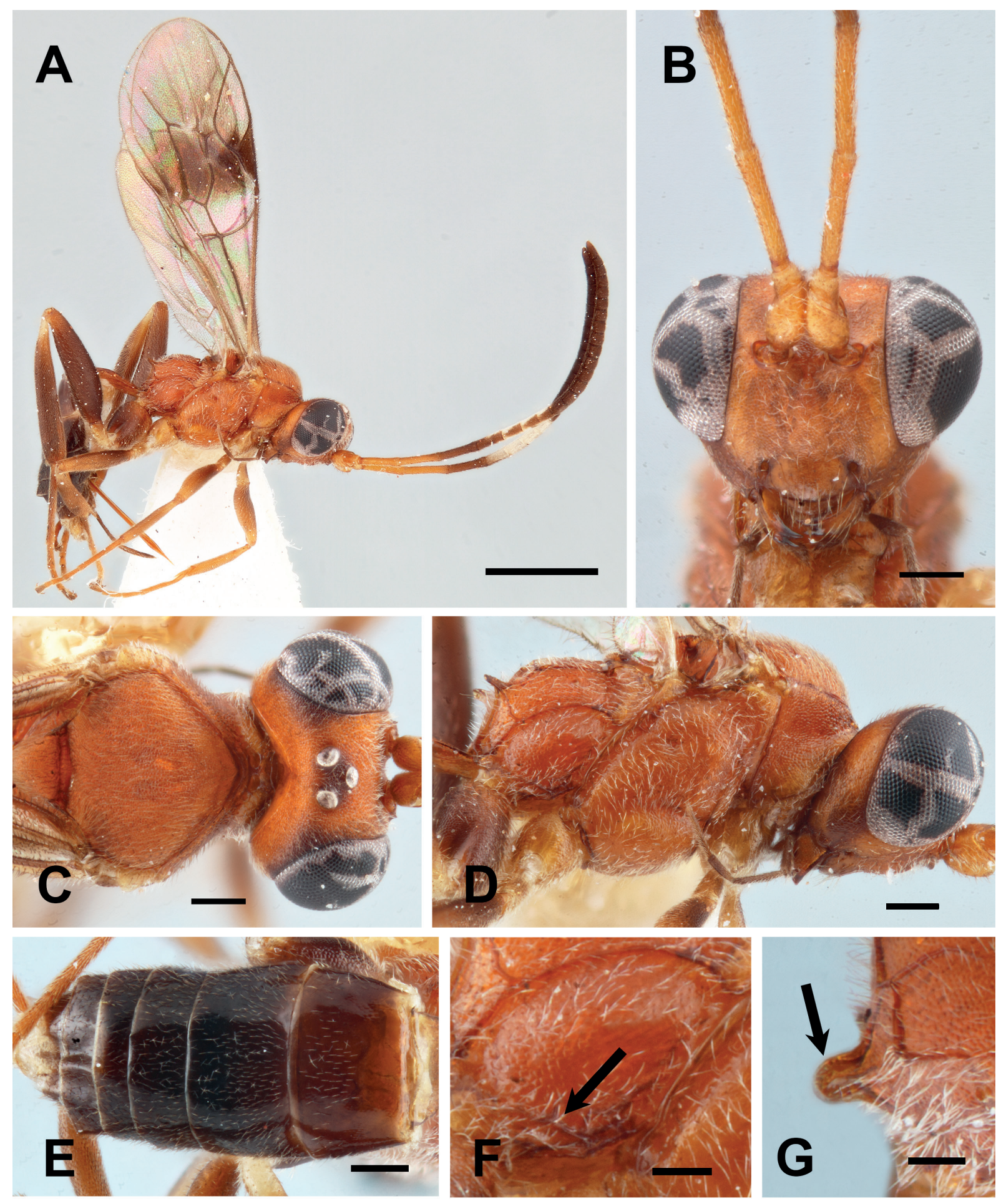

Fig. 4. Morphology of Handaoia mercedensis Bordera sp. nov., holotype, $q$ (CEUA). A. Habitus, lateral view. B. Head, front view. C. Head and mesoscutum, dorsal view. D. Head and mesosoma, lateral view. E. Metasoma, dorsal view. F. Metapleuron (arrow: juxtacoxal carina). G. Propodeum (arrow: propodeal apophysis). Scale bars: $A=1 \mathrm{~mm} ; \mathrm{B}-\mathrm{E}=0.2 \mathrm{~mm} ; \mathrm{F}-\mathrm{G}=0.1 \mathrm{~mm}$. 
HEAD. Finely granulate, matt, strongly narrowed behind eyes, occiput very concave centrally in dorsal view (Fig. 4C); gena $0.18 \times$ length of eye in dorsal view; posterior ocellus separated from eye by $1.3 \times$ its own maximum diameter; distance between posterior ocelli $0.9 \times$ maximum ocellar diameter (Fig. 4C); occipital carina gently V-shaped medially; malar space about $1.4 \times$ basal width of mandible; face slightly swollen medially; clypeus (Fig. 4B) quite convex, gently granulate, with scattered coarse punctures in apical half, apex slightly rounded, about $2.0 \times$ as wide as high; mandible quite tapered to apex, ventral tooth of mandible a little shorter than upper tooth; maxillary palp reaching mid coxa; flagellum widened in middle, tapered towards apex, flagellomeres 8-20 flattened ventrally (Fig. 4A); flagellum with 22 segments, length-thickness ratios: $1^{\text {st }}$ segment $=5.0,2^{\text {nd }}=5.38$ and $10^{\text {th }}=1.0$.

Mesosoma. Finely granulate and matt (Fig. 4C-D); mesoscutum slightly convex, notauli absent (Fig. 4C); scutellum moderately convex, without lateral carinae; sternaulus deep, exceeding half the length of mesopleuron (Fig. 4D); juxtacoxal carina strong, irregular, joining submetapleural carina at mid length (Fig. 4F, arrow); propodeum (Fig. 1C) with strong and conspicuous carinae; area superomedia confluent with area basalis and separated from area petiolaris; inner side of area externa about $4.7 \times$ as long as inner side of area dentipara; inner side of area dentipara about $0.3 \times$ length of outer side; area spiracularis confluent with area lateralis; posterior transverse carina strong, forming an elevate apophysis joining lateral longitudinal carina, apophysis parallel-sided with blut tip (Fig. 4G, arrow). Length of hind femur about $4.0 \times$ its height. Hind wing with vein $c u-a 0.5$ as long as abscissa of $C U$ between $M$ and $c u-a$.

Metasoma. Tergite I (Fig. 1C) finely granulate, slightly shiny, $1.77 \times$ as long as its maximum width; postpetiole laterally not finely strigose; lateromedian longitudinal carina moderately strong, reaching about $0.7 \times$ length of tergite; lateral longitudinal carina complete; tergite II (Fig. 4E) shiny, very slightly granulate anteriorly, glabrous, smooth posteriorly, with moderately dense short setae; remaining tergites (Fig. 4E) smooth and shiny with inconspicuous dense setiferous punctures. Ovipositor sheath $0.53 \times$ as long as hind tibia.

Colour. Head (Fig. 4A-D) orange brown, vertical orbits and interocellar area dark brown; palpi dark brown; mandibular teeth black; antenna with scape, pedicel and flagellomeres 1-2 light brown, remaining flagellomeres dark brown, flagellomeres 4-7 and base of 8 dorsally white (Fig. 4A). Mesosoma (Fig. 4A, C-D) entirely orange-brown. Metasoma (Fig. 4E) except tergite I dark brown, tergite I light brown. Fore and mid legs (Fig. 4A) light brown, tarsi fuscous; hind leg dark brown, trochanter and base of tibia white. Wings slightly infuscate, fore wing with well-defined transverse dark brown band (Fig. 4A).

\section{Male \\ Unknown.}

Handaoia plaumanni sp. nov. urn:1sid:zoobank.org:act:7A2E9032-B90C-4EB7-9D7F-139AA2D3D4B9

Figs $1 \mathrm{D}, 5-6,9 \mathrm{~B}$

\section{Diagnosis}

Handaoia plaumanni sp.nov. can be distinguished from all other New World species by the combination of the following characters (both male and female): apophysis of propodeum as a low transverse crest (Fig. 5I, arrow); sternaulus very short and weak, reaching at most $0.3 \times$ the length of the mesopleuron (Figs 5E, 6D); juxtacoxal carina absent (Figs 5H, 6E); area externa of propodeum confluent with area dentipara (Figs 1D, 6F). 


\section{Etymology}

This species is named in honour of Dr Fritz Plaumann, illustrious botanist and entomologist based in Brazil, who collected the type series. A noun in the genitive case.

\section{Material examined}

Known from three females and two males.

\section{Holotype}

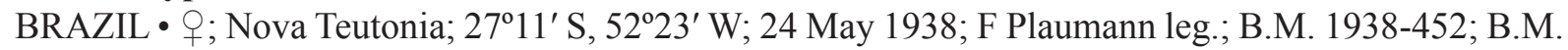
Type Hym 3b.2872; NHMUK010636386.

\section{Paratypes}

BRAZIL • 1 9; same collection data as for holotype; 13 Apr. 1938; B.M. 1938-682; NHMUK010636382 - 1 ठ̊; same collection data as for preceding; 10 May 1938; B.M. 1938-682; NHMUK010636384 • 1 \%; same collection data as for preceding; 13 May 1938; B.M. 1938-682; NHMUK010636395 • 1 §ै; same collection data as for preceding; 7 Jun. 1938; B.M. 1938-458; NHMUK010636385.

\section{Description}

\section{Female}

MeAsurements. Body length (without ovipositor) 2.6-2.9 mm. Fore wing 2.3-2.4 mm long.

HEAD. Moderately coarsely granulate, matt, distinctly narrowed behind eyes, occiput very concave centrally in dorsal view (Fig. 5B-E); gena $0.16-0.25 \times$ length of eye in dorsal view; posterior ocellus separated from eye by $0.9-1.0 \times$ its maximum diameter; distance between posterior ocelli $0.75-1.3 \times$ maximum ocellar diameter (Fig. 5D); occipital carina gently V-shaped medially (Fig. 5D); malar space $1.17-1.33 \times$ basal width of mandible; face weakly swollen medially (Fig. 5C); clypeus (Fig. 5C) weakly convex, smooth and shiny with scattered punctures, apex almost straight, about $2.0 \times$ as wide as high; mandible slightly tapered to apex, ventral tooth approximately same length as upper tooth; maxillary palp relatively short, reaching at most $2 / 3$ length of mesosternum; flagellum widened in middle, tapered towards apex, flagellomeres 8-17 flattened ventrally (Fig. 5A); flagellum with 18 segments, lengththickness ratios: $1^{\text {st }}$ segment $=4.67-5.0,2^{\text {nd }}=5.0-5.33$ and $10^{\text {th }}=1.0$.

Mesosoma. Moderately coarsely granulate, matt (Fig. 5D-E); mesoscutum slightly convex, flat posteriorly, notauli very weak anteriorly or absent (Fig. 5B, D), when present reaching at most 0.3 distance to scuto-scutellar groove; scutellum moderately convex, without lateral carinae; sternaulus very short and weak, as a wide V-shaped depression anteriorly, reaching at most $0.3 \times$ the length of mesopleuron (Fig. 5E); juxtacoxal carina absent (Fig. 5H); propodeum (Figs 1D, 5I) with moderately strong carinae; area superomedia confluent with area basalis and separated from area petiolaris; area externa and area dentipara confluent; area spiracularis confluent with area lateralis; posterior transverse carina forming a very low transverse crest joining lateral longitudinal carina (Fig. 5I, arrow). Length of hind femur 4.0-4.26 $\times$ its height. Hind wing with vein $c u-a 0.4-0.6 \times$ as long as abscissa of $C U$ between $M$ and $c u-a$.

Metasoma. Tergite I (Figs 1D, 5J) finely granulate, matt, $1.6-1.8 \times$ as long as its maximum width; lateromedian longitudinal carina absent; lateral longitudinal carina weak, absent posterior to spiracles; tergite II (Fig. 5F-G) smooth and shiny, glabrous; remaining tergites (Fig. 5F-G) smooth and shiny with inconspicuous dense setiferous punctures. Ovipositor sheath about $0.5-0.6 \times$ as long as hind tibia.

CoLour. Head (Fig. 5B-E) dark brown; antenna with scape and pedicel light brown and flagellomeres 1-4 light brown sometimes infuscate, remaining flagellomeres dark brown, flagellomeres 4-7 dorsally 

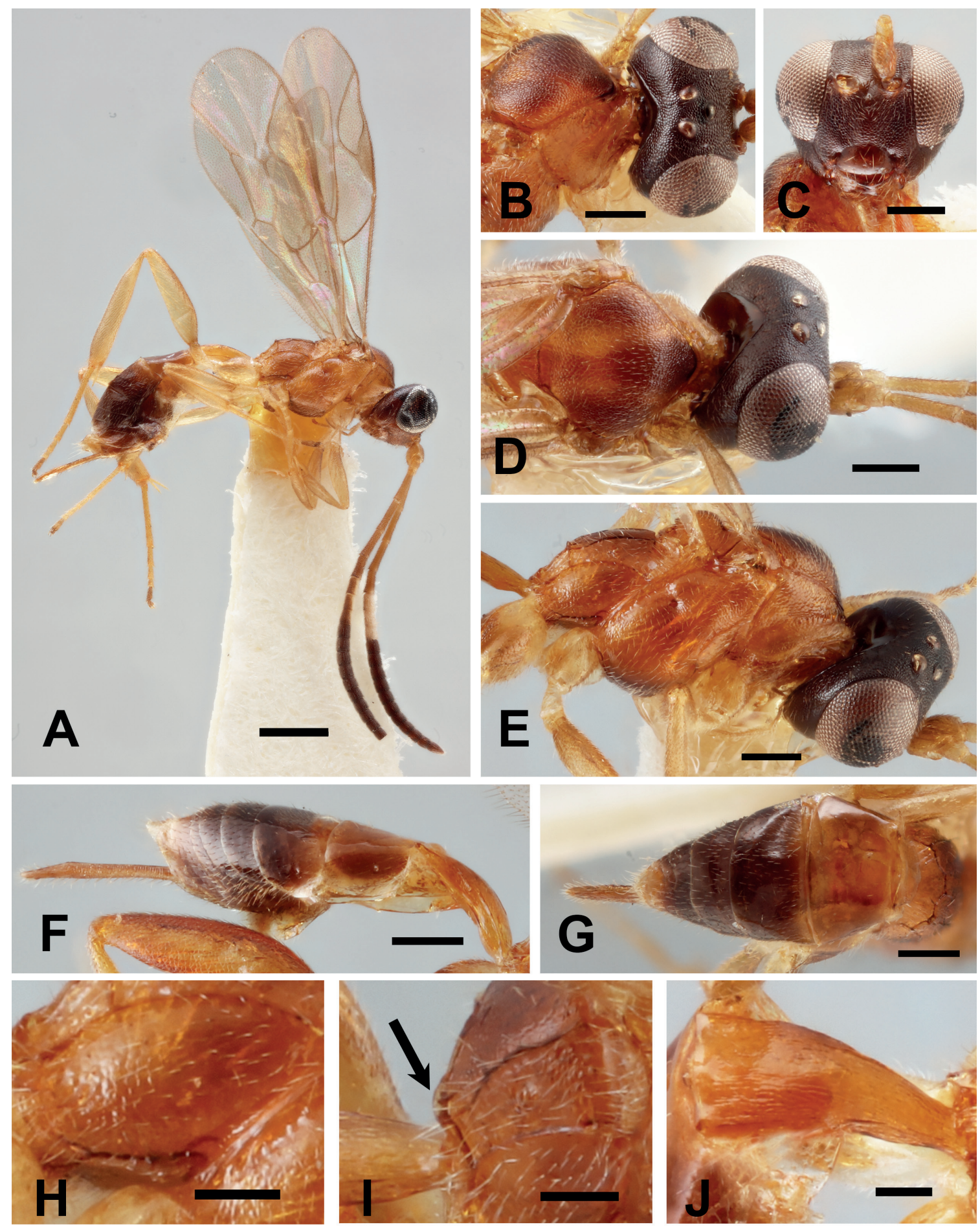

Fig. 5. Morphology of Handaoia plaumanni sp. nov. A. Habitus, lateral view, holotype, $q$ (NHMUK010636386). B. Head, dorsal view, paratype, o (NHMUK010636395). C. Head, front view, paratype, $q$ (NHMUK010636382). D. Mesoscutum, dorsal view, paratype, ㅇ (NHMUK010636395). E. Head and mesosoma, lateral view, paratype, $q$ (NHMUK010636395). F. Metasoma, lateral view, paratype, $q$ (NHMUK010636382). G. Metasoma, dorsal view, paratype, $q$ (NHMUK010636382). H. Metapleuron, paratype, + (NHMUK010636395). I. Propodeum, paratype, $q$ (NHMUK010636382) (arrow: propodeal apophysis). J. Tergite I, dorsal view, paratype, o (NHMUK010636382). Scale bars: $\mathrm{A}=0.5 \mathrm{~mm} ; \mathrm{B}-\mathrm{G}=0.2 \mathrm{~mm} ; \mathrm{H}-\mathrm{J}=0.1 \mathrm{~mm}$. 


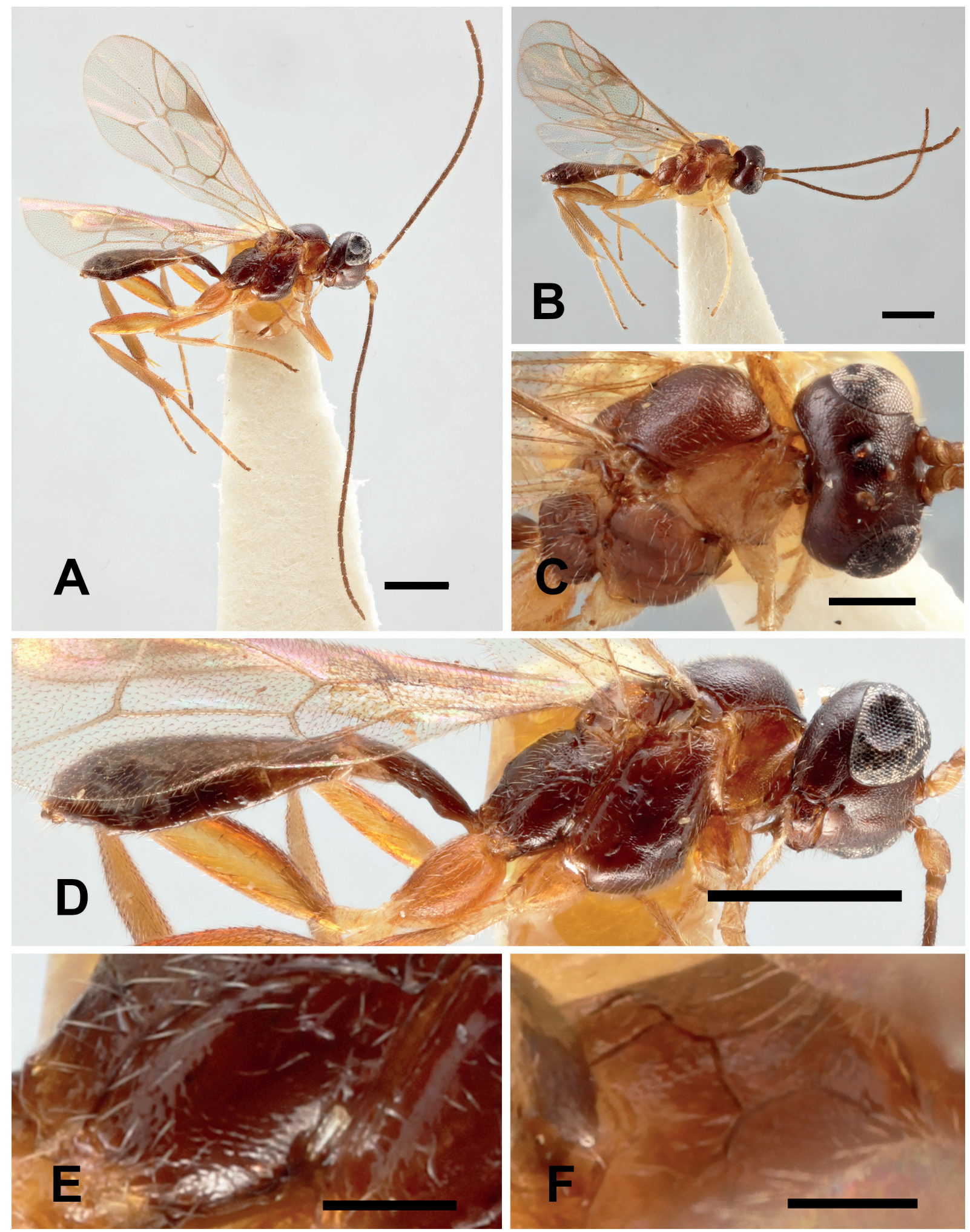

Fig. 6. Morphology of Handaoia plaumanni sp. nov. A. Habitus, lateral view, paratype, $\widehat{0}$ (NHMUK010636385). B. Habitus, lateral view, paratype, ô (NHMUK010636384). C. Head and mesosoma, dorsolateral view, paratype, §̊ (NHMUK010636384). D. Habitus, lateral view, paratype, $\widehat{\jmath}$ (NHMUK010636385). E. Metapleuron, paratype, ô (NHMUK010636385). F. Propodeum, dorsal view, paratype, $\hat{\jmath}$ (NHMUK010636384). Scale bars: A-B $=0.5 \mathrm{~mm}$; $\mathrm{C}-\mathrm{D}=0.2 \mathrm{~mm}$; $\mathrm{E}-\mathrm{F}=0.1 \mathrm{~mm}$. 
white (Fig. 5A). Mesosoma (Fig. 5B, D-E) mostly orange-brown; mesoscutum tending to entirely dark brown, sometimes anterior part of propodeum infuscate. Metasoma (Fig. 5F-G) brown, tergites I-II and sometimes anterior part of III light brown, remaining tergites dark brown. Legs (Fig. 5A) light brown, distal tarsomeres dark brown, sometimes fore and mid coxae, part of hind coxa and all trochanters and trochantelli lighter. Wings (Fig. 5A) slightly infuscate.

\section{Male}

Measurements. Body length 2.3-2.9 mm. Fore wing 2.0-2.6 mm long.

HEAD. Less narrowed behind eyes and less concave centrally in dorsal view than female (Fig. 6C); gena $0.31-0.42 \times$ length of eye in dorsal view; posterior ocellus separated from eye by $1.25-1.33 \times$ its maximum diameter; distance between posterior ocelli $0.9-1.3 \times$ maximum ocellar diameter (Fig. $6 \mathrm{C}$ ); malar space $1.33 \times$ basal width of mandible; clypeus about $1.7 \times$ as wide as high; flagellum filiform (Fig. 6A-B) with 19 segments, length-thickness ratios: $1^{\text {st }}=$ segment $5.0,2^{\text {nd }}=5.0$ and $10^{\text {th }}=3.4-4.0$

Mesosoma. Notauli absent (Fig. 6C-D); length of hind femur about 4.5-4.6 $\times$ its heigth.

Metasoma. Tergite I finely granulate, matt, $2.1 \times$ as long as its maximum width.

CoLour. As in female (Fig. 6A-F) but antenna entirely dark brown. In one paratype (NHMUK010636385), mesosoma and metasoma mostly dark brown (Fig. 6A, D).

Otherwise similar to female.

Handaoia ruizcancinoi Bordera sp. nov. urn:1sid:zoobank.org:act:151C284F-8024-4A2A-97FD-D0BBDE6AF048

Figs 1E, 7, 9A

\section{Diagnosis}

Handaoia ruizcancinoi Bordera sp. nov. can be distinguished from all other New World species by the combination of the following characters: apophysis of propodeum conspicuously high, subtriangular with blunt tip (Fig. 7H, arrow); juxtacoxal carina weak but complete, joining submetapleural carina at its anterior part (Fig. 7G, arrow); inner side of area externa about $7.0 \times$ as long as inner side of area dentipara (Fig. 1E).

\section{Etymology}

The species is named in honour of Dr Enrique Ruiz Cancino from Universidad Autónoma de Tamaulipas (Mexico), in recognition of his contribution to the study of the Ichneumonidae of Mexico. A noun in the genitive case.

\section{Material examined}

Known only from the holotype female.

\section{Holotype}

MEXICO • '; Tamaulipas, Ocampo, J. de Manantiales; T. Amarillas; 13 May 1995; J. Coronado, S. Nino and C. Hernández leg.; CEUA.

\section{Description}

Female

Measurements. Body length (without ovipositor) $4.0 \mathrm{~mm}$. Fore wing about $3.0 \mathrm{~mm}$ long. 


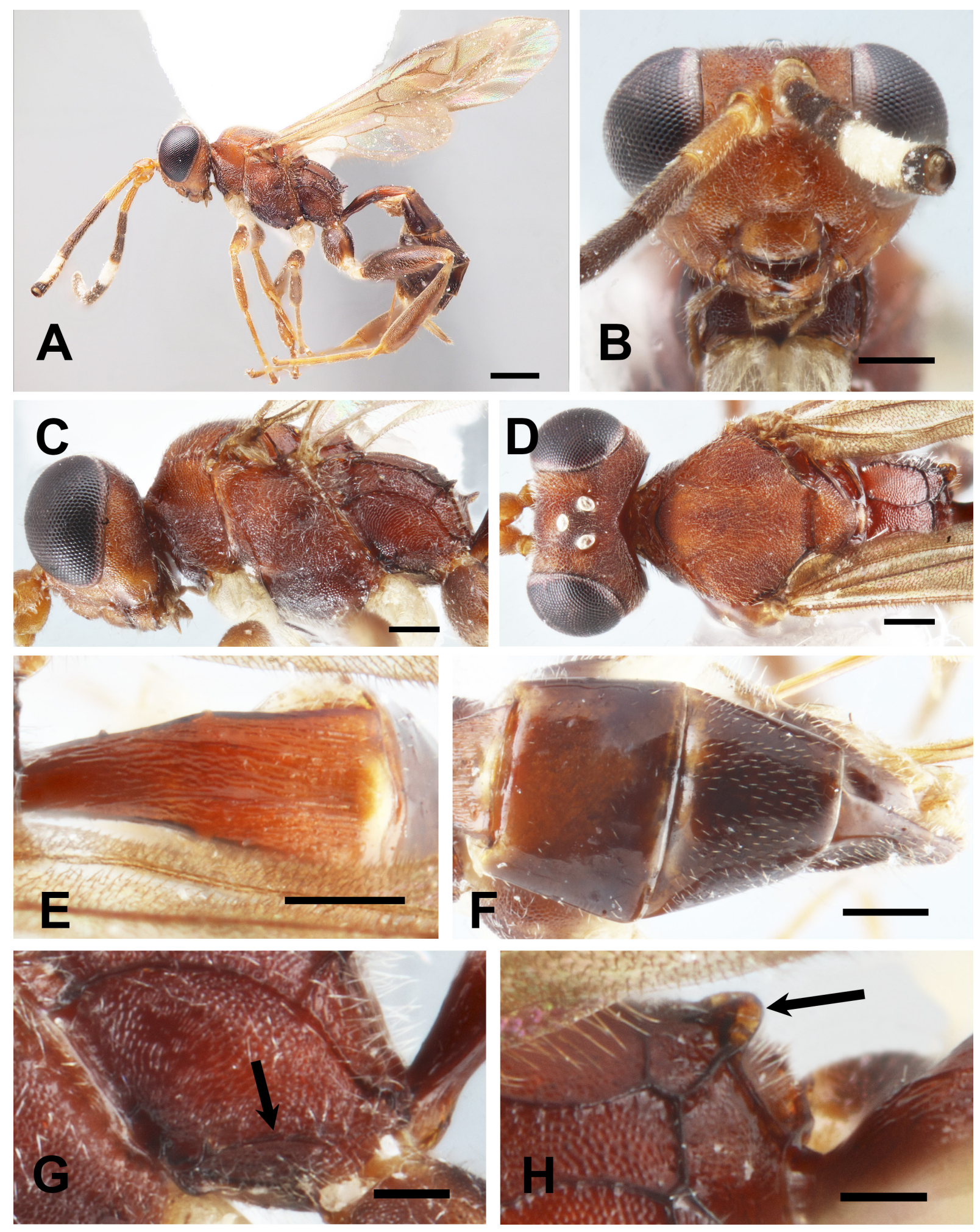

Fig. 7. Morphology of Handaoia ruizcancinoi Bordera sp. nov., holotype, $q$ (CEUA). A. Habitus, lateral view. B. Head, front view. C. Head and mososoma, lateral view. D. Head and mososoma, dorsal view. E. Tergite I, dorsal view. F. Metasoma, dorsal view. G. Metapleuron (arrow: juxtacoxal carina). H. Propodeum (arrow: propodeal apophysis). Scale bars: A $=0.5 \mathrm{~mm} ; \mathrm{B}-\mathrm{F}=0.2 \mathrm{~mm} ; \mathrm{G}-\mathrm{H}=0.1 \mathrm{~mm}$. 
HEAD. Finely granulate, matt, strongly narrowed behind eyes, occiput very concave centrally in dorsal view (Fig. 7B-D); gena $0.14 \times$ length of eye in dorsal view; posterior ocellus separated from eye by $1.55 \times$ its maximum diameter; distance between posterior ocelli $1.27 \times$ maximum ocellar diameter; occipital carina gently V-shaped medially (Fig. 7D); malar space $1.3 \times$ basal width of mandible; face conspicuously swollen medially (Fig. 7B); clypeus (Fig. 7B) quite convex, gently granulate with scattered coarse punctures, apex slightly rounded, about $1.8 \times$ as wide as high; mandible quite tapered to apex, ventral tooth of mandible a little shorter than upper tooth; flagellum widened in middle, tapered towards apex, flagellomeres 8-18 flattened ventrally; flagellum with 20 segments, length-thickness ratios: $1^{\text {st }}$ segment $=4.1,2^{\text {nd }}=3.27$ and $10^{\text {th }}=0.72$.

Mesosoma. Finely granulate and matt (Fig. 7A, C-D); mesoscutum slightly convex, almost flat posteriorly; notauli moderately deep over $0.7 \times$ length of mesoscutum (Fig. 7D); scutellum moderately convex, without lateral carinae; sternaulus as a wide V-shaped depression anteriorly, reaching about $0.3 \times$ the length of mesopleuron (Fig. 7C); juxtacoxal carina weak but complete, joining submetapleural carina at its anterior part (Fig. 7G, arrow); propodeum (Figs 1E, 7C, H) with strong and conspicuous carinae; area superomedia confluent with area basalis and separated from area petiolaris; inner side of area externa about $7.0 \times$ as long as inner side of area dentipara; inner side of area dentipara about $0.3 \times$ length of outer side; area spiracularis confluent with area lateralis; posterior transverse carina strong, forming an elevated apophysis joining lateral longitudinal carina, apophysis subtriangular with blunt tip (Fig. 7C, H, arrow). Length of hind femur $3.9 \times$ its height. Hind wing with vein $c u-a 0.77 \times$ as long as abscissa of $C U$ between $M$ and $c u-a$.

Metasoma. Tergite I (Fig. 7E) finely granulate, shiny, strigose laterally, about $2.4 \times$ as long as its maximum width; lateromedian longitudinal carina moderately strong, reaching posterior $0.8 \times$ length of tergite; lateral longitudinal carina present from spiracle to posterior margin of postpetiole; tergite II (Fig. 7F) shiny, smooth and almost glabrous; remaining tergites (Fig. 7F) smooth and shiny, with short and moderately dense setiferous punctures. Ovipositor sheath about $0.5 \times$ as long as hind tibia.

CoLour. Head (Fig. 7B-D) brown, darker dorsally, lighter ventrally, mandibular teeth and labrum dark brown; antenna with scape, pedicel and first flagellomere light brown, remaining flagellomeres dark brown, flagellomeres 5-7 dorsally white (Fig. 7A). Mesosoma (Fig. 7A, C) brown, more infuscate ventrally. Metasoma (Fig. 7E-F) dark brown. Legs (Fig. 7A) mostly dark brown; fore and mid coxae and trochanters, and hind trochanter, white. Wings (Fig. 7A) evenly infuscate.

\section{Male}

Unknown.

Handaoia urceus sp. nov. urn:1sid:zoobank.org:act:5413C7C8-D785-4583-803F-0C1E75B7EA9E

Figs 1F, 8, 9B

\section{Diagnosis}

Handaoia urceus sp. nov. can be distinguished from all other New World species by the combination of the following characters: apophysis of propodeum as a low transverse rounded crest (Fig. 8I, arrow); tergite II finely granulate (Fig. 8D); juxtacoxal carina strong anteriorly, weak posteriorly, joining submetapleural carina at its half length (Fig. $8 \mathrm{H}$, arrow); area externa of propodeum separated from area dentipara by strong carina (Fig. $1 \mathrm{~F}$ ); inner side of area externa about $8.0 \times$ as long as inner side of area dentipara (Fig. 1F). 


\section{Etymology}

From the Latin for 'pitcher', named after the flask- or pitcher-shaped combined area basalis + superomedia. A noun in apposition.

\section{Material examined}

Known only from the holotype female.

\section{Holotype}

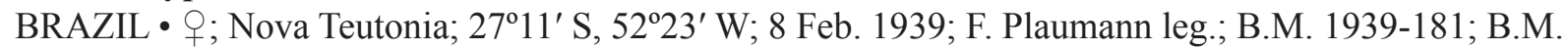
Type Hym 3b.2873; NHMUK010636380.

\section{Description}

\section{Female}

Measurements. Body length (without ovipositor) $4.3 \mathrm{~mm}$. Fore wing $3.7 \mathrm{~mm}$ long.

HEAD. Finely granulate, matt, distinctly narrowed behind eyes, occiput very concave centrally in dorsal view (Fig. 8B-C); gena $0.22 \times$ length of eye in dorsal view; posterior ocellus separated from eye by $1.0 \times$ its maximum diameter; distance between posterior ocelli $1.0 \times$ maximum ocellar diameter (Fig. 8C); occipital carina gently V-shaped medially (Fig. 8E); malar space $1.44 \times$ basal width of mandible; face moderately swollen medially (Fig. 8B); clypeus (Fig. 8B) weakly convex, gently granulate with scattered punctures, apex slightly rounded, about $1.6 \times$ as wide as high; mandible slightly tapered to apex, ventral tooth approximately same length as upper tooth; maxillary palp long, almost reaching middle coxa; flagellum widened in middle, tapered towards apex (Fig. 8A), flagellomeres $8-18$ flattened ventrally; flagellum with 22 segments, length-thickness ratios: $1^{\text {st }}$ segment $=4.9,2^{\text {nd }}=6.1$ and $10^{\text {th }}=1.13$.

Mesosoma. Finely granulate and matt (Fig. 8E, G); mesoscutum (Fig. 8E, G) convex, notauli moderately deep anteriorly, reaching about $0.3 \times$ distance to scuto-scutellar groove; scutellum moderately convex, without lateral carinae; sternaulus moderately deep, reaching about mid-point of mesopleuron (Fig. 8G); juxtacoxal carina strong anteriorly, weak posteriorly, joining submetapleural carina at mid length (Fig. $8 \mathrm{H}$, arrow); propodeum (Figs 1F, 8I) with strong and conspicuous carinae; area superomedia confluent with area basalis and separated from area petiolaris; inner side of area externa about $8.0 \times$ as long as inner side of area dentipara; inner side of area dentipara $0.25 \times$ length of outer side; area spiracularis confluent with area lateralis; posterior transverse carina strongly elevated, forming a low transverse crest joining lateral longitudinal carina (Fig. 8I, arrow). Length of hind femur $4.2 \times$ its height. Hind wing with vein $\mathrm{cu}-a$ about $1.0 \times$ as long as abscissa of $C U$ between $M$ and $c u-a$.

Metasoma. Tergite I (Figs 1F, 8D) finely granulate, matt, about $1.70 \times$ as long as its maximum width; postpetiole laterally and posteriorly tending to fine strigosity; lateromedian and lateral longitudinal carinae strong, reaching at least $0.8 \times$ length of tergite; tergite II (Fig. $8 \mathrm{D}, \mathrm{F}$ ) finely granulate, shiny; remaining tergites (Fig. 8D, F) smooth and shiny with inconspicuous setiferous punctures. Ovipositor sheath about $0.54 \times$ as long as hind tibia.

Colour. Head (Fig. 8B-C) dark brown tending to be more reddish on clypeus and mandibles; palpi light brown; antenna with scape, pedicel and flagellomeres I-II (III) light brown, remaining flagellomeres dark brown, distal part of flagellomere 4 to flagellomere 7 dorsally white (Fig. 8A). Mesosoma (Fig. 8EG) entirely orange. Metasoma (Fig. 8D-F) with a gradient from orange at anterior of tergite I to dark brown on posterior tergites, posterior rim of tergites I-III yellowish. Legs (Fig. 8A) light brown, distal tarsomeres dark brown, fore and mid coxae, part of hind coxa and all trochanters and trochantelli lighter. Wings (Fig. 8A) slightly infuscate. 

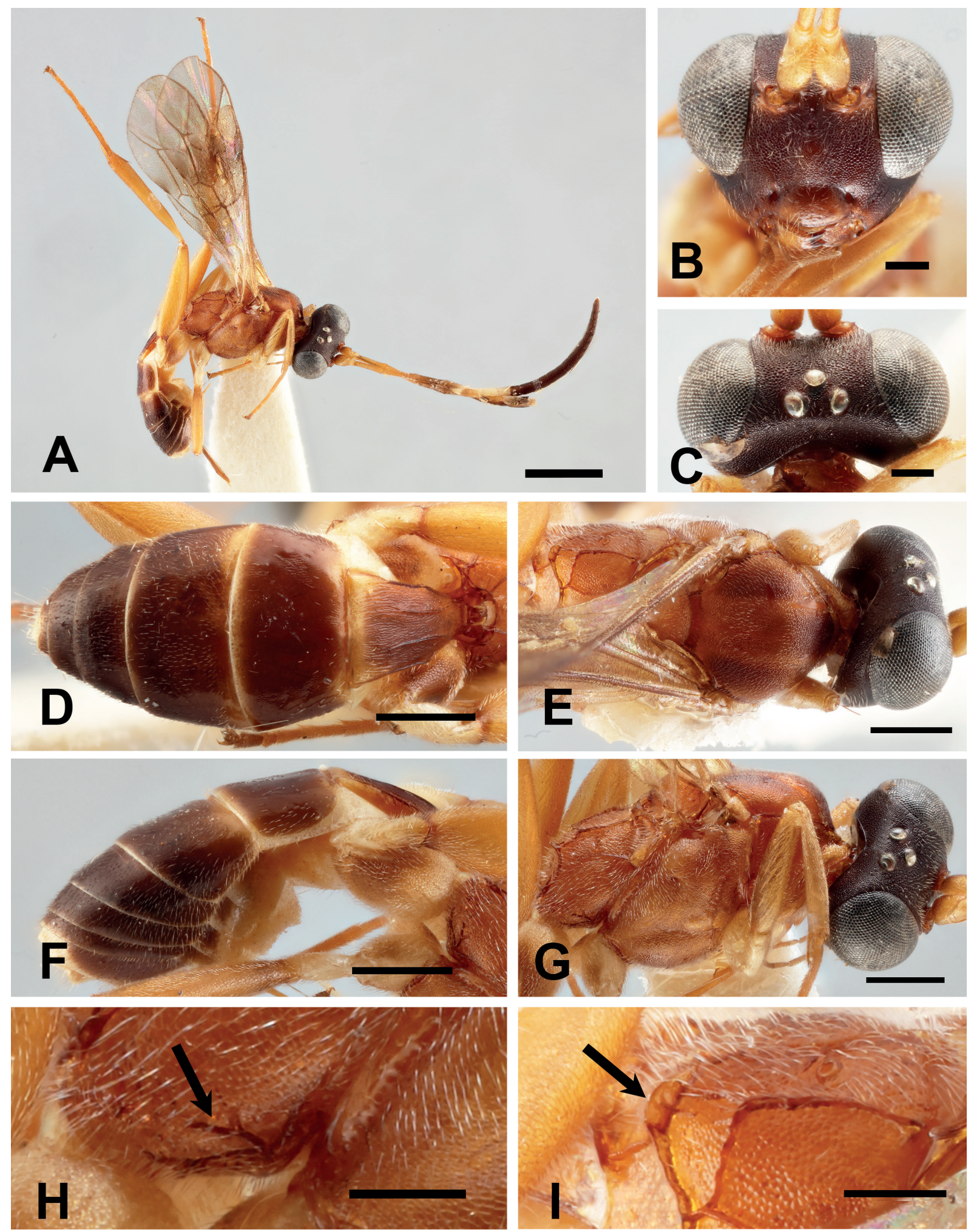

Fig. 8. Morphology of Handaoia urceus sp. nov., holotype, $q$ (NMHUK). A. Habitus, lateral view. B. Head, front view. C. Head, dorsal view. D. Metasoma, dorsal view. E. Head and mesosoma, dorsal view. F. Metasoma, lateral view. G. Head and mesosoma, dorsolateral view. H. Metapleuron (arrow: juxtacoxal carina). I. Propodeum (arrow: propodeal apophysis). Scale bars: A, D-G $=0.5 \mathrm{~mm}$; B-C, $\mathrm{H}-\mathrm{I}=0.2 \mathrm{~mm}$. 
Male

Unknown.

\section{Discussion}

This study significantly improves our knowledge of Neotropical Handaoia, previously only known from two undescribed Peruvian species (Townes 1970), to the recognition of six new species from widely scattered locations in Brazil, Mexico and Peru. However, there are undoubtedly more species to be discovered. There are many Neotropical phygadeuontines in collections that have yet to be identified to genus; sorting and identifying specimens is obviously essential to make these accessible, but this is a task that requires available expertise in ichneumonid identification, which is often a severely limiting factor.

The classification of Handaoia requires further discussion. Although Townes (1970) included Handaoia in his 'Chiroticina' subtribe of what is now the subfamily Phygadeuontinae (Santos, 2017), the few phylogenetic analyses of Phygadeuontinae have found that the chiroticine genera do not form a clade (Laurenne et al. 2006; Quicke et al. 2009; Santos 2017). In fact, there is little evidence for the monophyly of most subtribes in Phygadeuontinae or Cryptinae Kirby, 1837. In a combined morphological and molecular analysis (although with all Phygadeuontinae coded identically), Quicke et al. (2009) found that Handaoia and also Austriteles Gauld, 1984 formed a clade with Ateleute Förster, 1869 and Tamaulipeca Kasparyan, 2000, two of the three genera comprising the recently recognised Ateleutinae Townes, 1970 (Santos 2017; Santos et al. 2018). Could these genera be better classified in Ateleutinae? The morphological evidence is weak: Handaoia shares the complete posterior carina of the mesosternum and a single large bulla in fore wing vein $2 \mathrm{~m}-\mathrm{cu}$ with Ateleutinae; Austriteles shares the single bulla and a first metasomal segment with the spiracle at about mid-length and the sternite ending anterior to the spiracle. There are also numerous differences between the currently included genera within the Ateleutinae
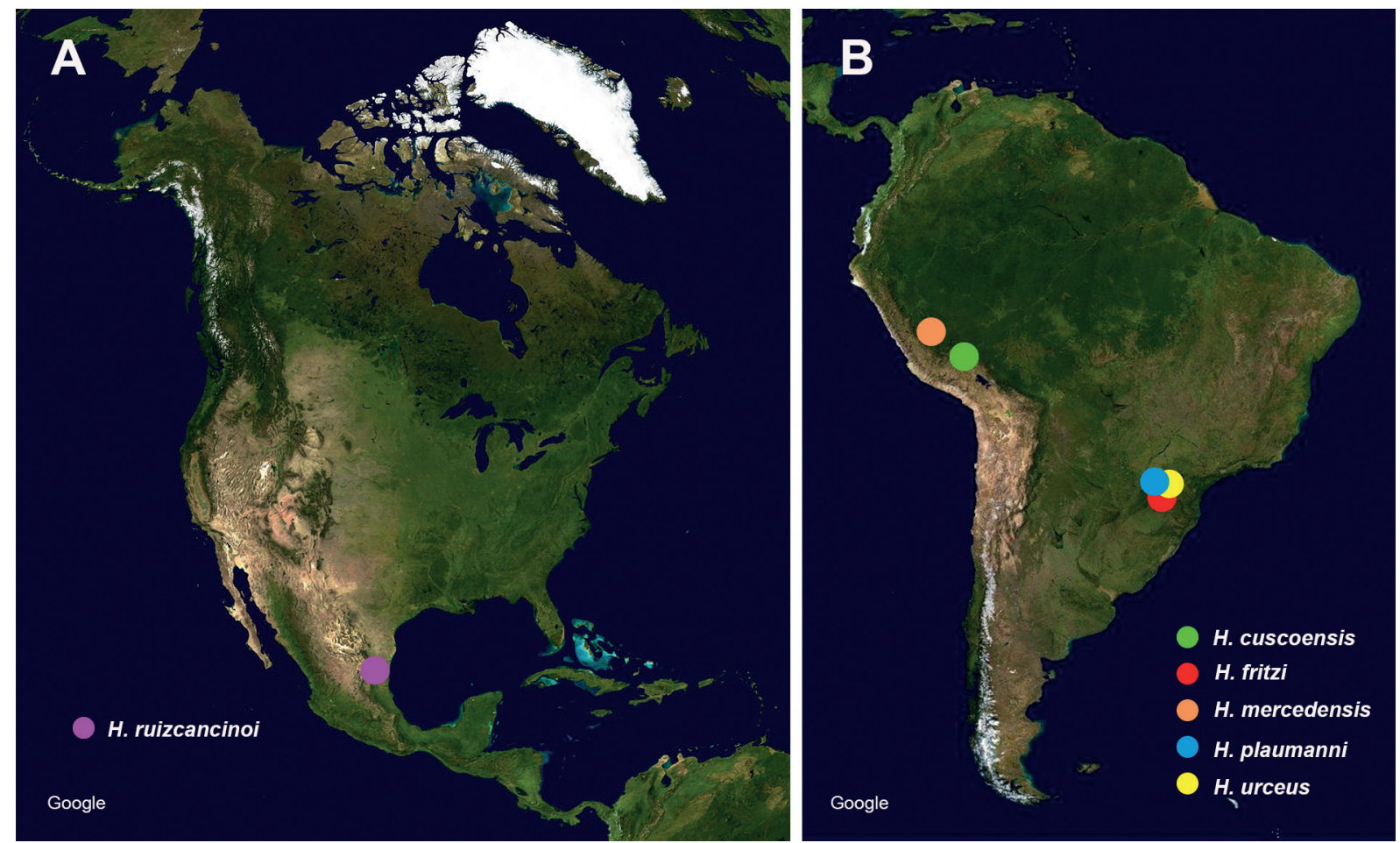

Fig. 9. Distribution of New World species of Handaoia Seyrig, 1952. A. North and Central America. B. South America. 
and both Handaoia and Austriteles. It would be well worth sequencing additional genes (Quicke et al. 2009 only sequenced one gene) and more thoroughly evaluating the potential synapomorphies of an expanded Ateleutinae. We also take the opportunity to note that the Australian species that Gauld (1984) assigned to Handaoia differ markedly from other Handaoia: the propodeum of the Australian species has the transverse carinae strong, with the area superomedia basically absent, and the dorsal pronotal groove simple. These might be better reclassified in another genus.

We have not examined specimens of other species of Handaoia but we have seen high quality photographs of type material of $H$. arenacea Seyrig, 1952, H. brevipennis Seyrig, 1952, H. elongata Seyrig, 1952, H. flexibilis Seyrig, 1952 and H. gracilior Seyrig, 1952 from the Paris Museum of Natural History (https://science.mnhn.fr/taxon/genus/handaoia) and of Hemiteles bellicornis Thomson, 1888 in the Biological Museum, Lund University (photos on Flickr: https://www.flickr.com/). The species we include in Handaoia are from widely scattered parts of the world, but they share a distinctive suite of characters, unique in combination, so we include them all in Handaoia. Handaoia bellicornis and the unidentified specimen illustrated in Townes (1970) have the area superomedia separated from the area basalis, whereas the Malagasy and New World species have these areas confluent. Without checking specimens it is difficult to evaluate the presence or absence of the carina across the dorsal pronotal groove, which seems to be absent in some species.

\section{Acknowledgements}

The authors are grateful to Dr David Wahl from AEIC for permitting us to study the material preserved in his institution. We sincerely appreciate the collaboration of Dr Alexander R. Rodríguez Berrío (Universidad Nacional Agraria la Molina, Lima, Peru) who collected and provided the material of H. cuscoensis Bordera sp. nov. in the framework of the Project A/013484/07 from Agencia Española de Cooperación Internacional para el Desarrollo (Ministerio de Asuntos Exteriores y de Cooperación, Spain). Our special thanks to Dr Enrique Ruíz Cancino from Universidad Autónoma de Tamaulipas (Ciudad Victoria, Mexico), who kindly donated the material of $H$. ruizcancinoi Bordera sp. nov. to the CEUA. This study has been supported by a grant from the University of Alicante, Alicante, Spain to S. Bordera to visit NHMUK (Programa Propi del Vicerectorat d'Investigació i Transferència del Coneiximent per al Foment de la I+D+I, ACIE19-03).

\section{References}

Broad G.R., Shaw M.R. \& Fitton M.G. 2018. The ichneumonid wasps of Britain and Ireland (Hymenoptera: Ichneumonidae): their classification and biology. Handbooks for the Identification of British Insects 7 (12): 1-418.

Eady R.D. 1968. Some illustrations of microsculpture in the Hymenoptera. Proceedings of the Royal Entomological Society of London (A) 43: 66-72. https://doi.org/10.1111/j.1365-3032.1968.tb01029.x

Gauld I.D. 1984. An Introduction to the Ichneumonidae of Australia. British Museum (Natural History), London.

Horstmann K. 1979. A revision of the types of the Hemiteles spp. described by Thomson (Hymenoptera: Ichneumonidae). Entomologica Scandinavica 10: 297-302. https://doi.org/10.1163/187631279X00187

Horstmann K. 1981. Typen revision der von Karl Hedwig beschriebenen Arten und Formen der Familie Ichneumonidae (Hymenoptera). Entomologische Mitteilungen aus dem Zoologischen Museum Hamburg 7: 65-82.

Laurenne N.M., Broad G.R. \& Quicke D.L.J. 2006. Direct optimization and multiple alignment of 28S D2D3 rDNA sequences: problems with indels on the way to amolecular phylogeny of the cryptine ichneumon wasps(Insecta:Hymenoptera).Cladistics22:442-473.https://doi.org/10.1111/j.1096-0031.2006.00112.x 
Quicke D.L.J., Laurenne N.M., Fitton M.G. \& Broad G.R. 2009. A thousand and one wasps: a 28S rDNA and morphological phylogeny of the Ichneumonidae (Insecta: Hymenoptera) with an investigation into alignment parameter space and elision. Journal of Natural History 43: 1305-1421.

https://doi.org/10.1080/00222930902807783

Santos B. 2017. Phylogeny and reclassification of Cryptini (Hymenoptera, Ichneumonidae, Cryptinae), with implications for ichneumonid higher-level classification. Systematic Entomology 42: 650-676. https://doi.org/10.1111/syen.12238

Santos B.F., Alvarado M., Sääksjärvi I.E., Van Noort S., Villemant C. \& Brady S.G. 2018. Molecular phylogeny of Ateleutinae (Hymenoptera: Ichneumonidae): systematics and biogeography of a widespread parasitoid wasp lineage. Zoological Journal of the Linnean Society 185: 1057-1078. https://doi.org/10.1093/zoolinnean/zly072

Seyrig A. 1952. Les Ichneumonides de Madagascar. IV Ichneumonidae Cryptinae. Mémoires de l'Académie malgache. Fascicule XIX: 1-213.

Szépligeti G. 1910. Hymenoptera. 3. Braconidae \& Ichneumonidae. In: Sjöstedt Y. (ed.) Wissenschaftliche Ergebnisse der Schwedischen Zoologischen Expedition nach dem Kilimandjaro, dem Meru und den umgebenden Massaisteppen Deutsch-Ostafrikas Band 2 [1910]: 25-96. P. Palmquists Aktiebolag, Stockholm. https://doi.org/10.5962/bhl.title.1805

Townes H. 1970. Genera of Ichneumonidae, Part 2. Memoirs of the American Entomological Institute 12: $1-537$.

Yu D.S., van Achterberg C. \& Horstmann K. 2016. World Ichneumonoidea 2015. Taxonomy, Biology, Morphology and Distribution. Taxapad Interactive Catalogue. Database on flash-drive. Nepean, Ottawa, Canada.

Manuscript received: 15 December 2020

Manuscript accepted: 1 May 2021

Published on: 30 June 2021

Topic editor: Nesrine Akkari

Desk editor: Eva-Maria Levermann

Printed versions of all papers are also deposited in the libraries of the institutes that are members of the EJT consortium: Muséum national d'histoire naturelle, Paris, France; Meise Botanic Garden, Belgium; Royal Museum for Central Africa, Tervuren, Belgium; Royal Belgian Institute of Natural Sciences, Brussels, Belgium; Natural History Museum of Denmark, Copenhagen, Denmark; Naturalis Biodiversity Center, Leiden, the Netherlands; Museo Nacional de Ciencias Naturales-CSIC, Madrid, Spain; Real Jardín Botánico de Madrid CSIC, Spain; Zoological Research Museum Alexander Koenig, Bonn, Germany; National Museum, Prague, Czech Republic. 